\title{
Numerical study of thermal radiation and mass transfer effects on free convection flow over a moving vertical porous plate using cubic B-spline collocation method
}

\author{
M. Abu zeid ${ }^{1}$, Khalid K. Ali ${ }^{2}$, M. A. Shaalan ${ }^{1 *}$ (D) and K. R. Raslan ${ }^{2}$
}

* Correspondence: abozeid87@ yahoo.com

${ }^{1}$ Basic Science Department, Higher Technological Institute, Tenth of Ramadan City, Egypt

Full list of author information is available at the end of the article

\begin{abstract}
In this paper, we present a numerical method based on cubic B-spline function for studying the effects of thermal radiation and mass transfer on free convection flow over a moving vertical porous plate. Similarity transformations reduced the governing partial differential equations of the fluid flow to a system of nonlinear ordinary differential equations which are solved numerically using a cubic B-spline collocation method. The effects of various physical parameters on the velocity, temperature, and concentration distributions are shown graphically, and the numerical values of physical quantities like skin friction, Nusselt number, and Sherwood number for various parameters are presented in tabular form and discussed.
\end{abstract}

Keywords: Cubic B-spline function, Thermal radiation, Mass transfer, Porous plate 2010 Mathematics Subject Classification: 41A15, 76Sxx, 80A20

\section{Introduction}

Free convection flow is important in many practical applications including cooling of electronic components, designs related to thermal insulation, material processing, and geothermal systems. The flow through porous media is important in many applications in science and engineering. Mixed convective flow past a semi-infinite vertical plate embedded in a porous medium incorporating the variable permeability in Darcy's model studied by Mohammadein and El-Shaer [1]. Zhang et al. [2] presented transient and steady natural convection from a heat source embedded in a saturated porous layer. The effects of Dufour and Soret numbers on steady combined free-forced convective and mass transfer flow past a semi-infinite vertical flat plate in the presence of a uniform transverse magnetic field studied by Alam et al. [3]. Brewster studied the properties of thermal radiative transfer [4]. Bestman examined the natural convection boundary layer with suction and mass transfer in a porous medium [5]. Singh and Soundalgekar investigated the transient free convection in cold water past an infinite porous plate [6]. Hossain studied the effect of transpiration on combined heat and mass transfer in mixed convection along a vertical plate [7]. Makinde studied free

(c) The Author(s). 2019 Open Access This article is distributed under the terms of the Creative Commons Attribution 4.0 International License (http://creativecommons.org/licenses/by/4.0/), which permits unrestricted use, distribution, and reproduction in any medium, provided you give appropriate credit to the original author(s) and the source, provide a link to the Creative Commons license, and indicate if changes were made. 
convection flow with thermal radiation and mass transfer past a moving vertical porous plate [8]. Reddy et al. studied radiation and chemical reaction effects on MHD flow along a moving vertical porous plate [9]. Reddy et al. [10] studied the mass transfer effects on unsteady hydromagnetic free convective memory flow of incompressible and electrically conducting fluid flow past an infinite vertical plate through a porous medium. Heat transfer by natural convection from a vertical flat plate embedded in electrically conducting micropolar fluid-saturated porous medium using the DarcyBrinkman-Forchheimer Boussinesq model in the presence of uniform magnetic field and thermal radiation was studied by Pal and Chatterjee [11]. Sharma et al. studied buoyancy effects on MHD unsteady convection of a radiating chemically reacting fluid past a moving porous vertical plate in a binary mixture [12]. Makinde et al. presented unsteady convection with chemical reaction and radiative heat transfer past a flat porous plate moving through a binary mixture [13]. Sajid et al. studied Darcy-Forchheimer flow of Maxwell nanofluid flow with nonlinear thermal radiation and activation energy [14]. Makinde and Olanrewaju presented unsteady mixed convection with Soret and Dufour effects past a porous plate moving through a binary mixture of chemically reacting fluid [15]. Makinde and Ogulu studied the effect of thermal radiation on the heat and mass transfer flow of a variable viscosity fluid past a vertical porous plate permeated by a transverse magnetic field [16]. Ogulu and Makinde presented unsteady hydromagnetic free convection flow of a dissipative and radiating fluid past a vertical plate with constant heat flux [17]. Caglar used the cubic B-spline for solving linear system of second-order boundary value problems [18]. This paper is organized as follows: In the section 2 , we analyze the mathematical problem. In the section 3 , we apply the cubic B-spline method. In the section 4, we investigate the numerical results and discussion. Finally, the conclusion of this study is given in the section 5 .

\section{Mathematical analysis}

Consider unsteady flow and free convection heat and mass transfer of incompressible viscous fluid past a moving vertical porous plate. Let $u$ and $v$ be the velocity components along the $x$ and the $y$ directions, respectively. We choose the $x$ axis that runs along the plate in the vertically upward direction and the $y$ axis be taken perpendicular to it as shown in Fig. 1. The physical variables are functions of $y$ and $t$ only.

According to Boussinesq's approximation, the governing equations that describe the physical situation can be written as:

$$
\begin{aligned}
& \frac{\partial v}{\partial y}=0 \\
& \frac{\partial u}{\partial t}+v \frac{\partial u}{\partial y}=v \frac{\partial^{2} u}{\partial y^{2}}+g \beta\left(T-T_{\infty}\right)+g \lambda\left(C-C_{\infty}\right), \\
& \frac{\partial T}{\partial t}+v \frac{\partial T}{\partial y}=\alpha \frac{\partial^{2} T}{\partial y^{2}}-\frac{\alpha}{k} \frac{\partial q_{r}}{\partial y} \\
& \frac{\partial C}{\partial t}+v \frac{\partial C}{\partial y}=D \frac{\partial^{2} C}{\partial y^{2}}
\end{aligned}
$$

with boundary conditions 


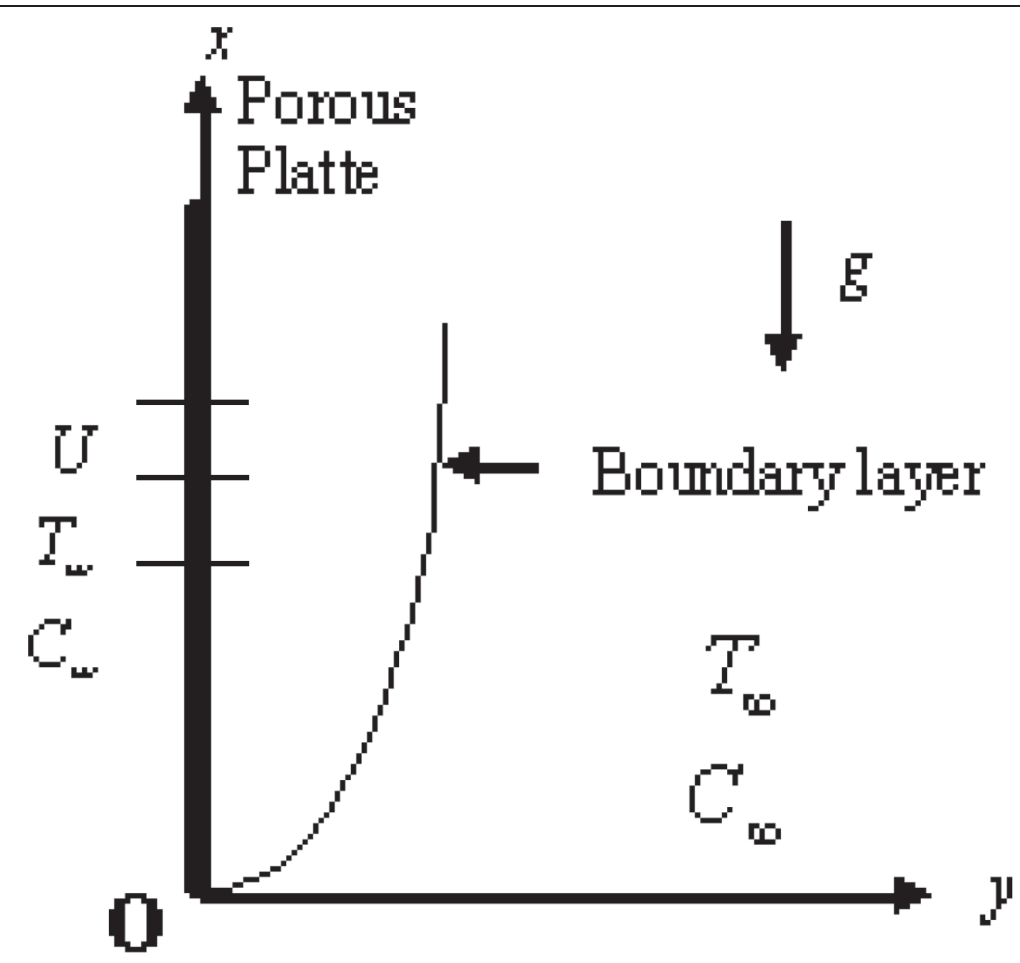

Fig. 1 The graphical abstract of the problem

$$
\begin{aligned}
& u=U, T=T_{w}, C=C_{w} \text { at } y=0, \\
& u \rightarrow 0, T \rightarrow T_{\infty}, C=C_{\infty} \text { as } y \rightarrow \infty \text { for } t>0,
\end{aligned}
$$

where $v$ is the kinematic viscosity, $g$ the acceleration due to gravity, $T$ the temperature, $C$ the concentration, $D$ the coefficient of mass diffusivity, $\alpha$ the thermal diffusivity, $k$ the thermal conductivity, $q_{r}$ the local radiative heat flux, $\beta$ volumetric expansion coefficient due to temperature, $\lambda$ volumetric expansion coefficient due to concentration, $T_{w}, C_{w}$ are the uniform temperature and concentration of the fluid at the plate, respectively, $T_{\infty}, C_{\infty}$ are the temperature and concentration of the fluid far away from the plate, respectively, and $U$ is the plate characteristic velocity. Using the Rosseland approximation [9], the radiative heat flux term is

$$
q_{r}=-\frac{4 \sigma}{3 \delta} \frac{\partial T^{4}}{\partial y}
$$

where $\sigma$ is the Stefan-Boltzmann constant and $\delta$ the mean absorption coefficient. We introduce similarity variables and the dimensionless quantities,

$$
\begin{aligned}
& \eta=\frac{y}{2 \sqrt{v t}}, u=U f(\eta), \theta=\frac{T-T_{\infty}}{T_{w}-T_{\infty}}, R a=\frac{16 \sigma\left(T_{w}-T_{\infty}\right)^{3}}{3 \delta k}, \\
& S c=\frac{v}{D}, \Phi=\frac{C-C_{\infty}}{C_{w}-C_{\infty}}, \operatorname{Pr}=\frac{v}{\alpha}, G_{r}=\frac{4 g \beta t\left(T_{w}-T_{\infty}\right)}{U}, \\
& G_{c}=\frac{4 g \lambda t\left(C_{w}-C_{\infty}\right)}{U}, N=\frac{T_{\infty}}{T_{w}-T_{\infty}}
\end{aligned}
$$

From Eq. (1), $v$ is either constant or a function of time. From [3], we choose 


$$
v=-c \sqrt{\frac{v}{t}}
$$

where $c>0$ is the suction parameter. Then, from Eqs. (8) and (9) in Eqs. (2), (3), and (4), we obtain system of ordinary differential equations as follows:

$$
\begin{aligned}
& f^{\prime \prime}+2(\eta+c) f^{\prime}=-G_{r} \theta-G_{c} \Phi \\
& \theta^{\prime \prime}+2(\eta+c) \operatorname{Pr} \theta^{\prime}=-R a\left(3(N+\theta)^{2} \theta^{\prime 2}+(N+\theta)^{3} \theta^{\prime \prime}\right), \\
& \Phi^{\prime \prime}+2(\eta+c) S c \Phi^{\prime}=0
\end{aligned}
$$

with the boundary conditions

$$
\begin{aligned}
& f(0)=\theta(0)=\Phi(0)=1, \\
& f(\infty)=\theta(\infty)=\Phi(\infty)=0,
\end{aligned}
$$

where $G_{r}$ and $G_{c}$ are the local free convection parameter, Pr is the Prandtl number, $\mathrm{Ra}$ is the radiation parameter, $N$ is the temperature difference parameter, and $S c$ is the Schmidt number.

\section{Cubic B-spline collocation method [12]}

The cubic B-splines $B_{i}(\eta)$ are given by

$$
B_{i}(\eta)=\frac{1}{h^{3}}\left\{\begin{array}{cc}
\left(\eta-\eta_{i-2}\right)^{3}, & \eta \in\left[\eta_{i-2}, \eta_{i-1}\right], \\
h^{3}+3 h^{2}\left(\eta-\eta_{i-1}\right)+3 h\left(\eta-\eta_{i-1}\right)^{2}-3\left(\eta-\eta_{i-1}\right)^{3}, & \eta \in\left[\eta_{i-1}, \eta_{i}\right] \\
h^{3}+3 h^{2}\left(\eta_{i+1}-\eta\right)+3 h\left(\eta_{i+1}-\eta\right)^{2}-3\left(\eta_{i+1}-\eta\right)^{3}, & \eta \in\left[\eta_{i}, \eta_{i+1}\right] \\
\left(\eta_{i+2}-\eta\right)^{3} & \eta \in\left[\eta_{i+1}, \eta_{i+2}\right], \\
0, & \text { otherwise } \\
(i=-1,0,1, \cdots, n+1), &
\end{array}\right.
$$

where $h=\eta_{i+1}-\eta_{i}, i=0,1, \ldots, n$ the values of the cubic B-spline $B_{i}(\eta)$ and all its first and second derivatives vanish outside the interval $\left(\eta_{i-2}, \eta_{i+2}\right)$ We establish the values of $B_{i}(\eta)$ and its derivatives at the knots $\eta_{i-2}, \eta_{i-1}, \ldots, \eta_{i+2}$ in Table 1 as:

We consider the cubic B-spline function to the solutions of the problems (10)-(12) as follows:

Table 1 The values of $B_{i}(\eta), B_{i}^{\prime}(\eta)$, and $B_{i}^{\prime \prime}(\eta)$

\begin{tabular}{llllll}
\hline & $\eta_{i-2}$ & $\eta_{i-1}$ & $\eta_{i}$ & $\eta_{i+1}$ & $\eta_{i+2}$ \\
\hline$B_{i}(\eta)$ & 0 & 1 & 4 & 1 & 0 \\
$B_{i}^{\prime}(\eta)$ & 0 & $-3 / h$ & 0 & $3 / h$ & 0 \\
$B_{i}^{\prime \prime}(\eta)$ & 0 & $6 / h^{2}$ & $-12 / h^{2}$ & $6 / h^{2}$ & 0 \\
\hline
\end{tabular}




$$
\left.\begin{array}{c}
f(\eta)=\sum_{i=-1}^{n+1} d_{i}(\eta) B_{i}(\eta), \\
\theta(\eta)=\sum_{i=-1}^{n+1} q_{i}(\eta) B_{i}(\eta), \\
\Phi(\eta)=\sum_{i=-1}^{n+1} s_{i}(\eta) B_{i}(\eta),
\end{array}\right\}
$$

where constants $d_{i}(\eta), q_{i}(\eta)$, and $s_{i}(\eta)$ 's are to be determined from the collocation points $\eta_{i}, i=0,1, \ldots, n$ and the boundary conditions.

Using Eq. (15) and the cubic B-spline $B_{i}(\eta)$ given by Eq. (14) and its principle twice derivatives $B_{i}^{\prime}(\eta)$ and $B_{i}^{\prime \prime}(\eta)$ at the knots and their values are summarized in Table 1, the values of $f_{i}, \theta_{i}, \Phi_{i}$ and their derivatives up to second order at the knots are

$$
\left.\begin{array}{rl}
f_{i} & =d_{i-1}+4 d_{i}+d_{i+1} \\
f_{i}^{\prime} & =-\frac{3}{h} d_{i-1}+\frac{3}{h} d_{i+1} \\
f_{i}^{\prime \prime} & =\frac{6}{h^{2}} d_{i-1}-\frac{12}{h^{2}} d_{i}+\frac{6}{h^{2}} d_{i+1}
\end{array}\right\}, i=0,1, \ldots, n .
$$

Substituting from Eqs. (16)-(18) in Eqs. (10)-(12), we find

$$
\begin{aligned}
& \frac{6}{h^{2}} d_{i-1}-\frac{12}{h^{2}} d_{i}+\frac{6}{h^{2}} d_{i-1}+2(i h+c)\left(-\frac{3}{h} d_{i-1}+\frac{3}{h} d_{i+1}\right) \\
& =-G_{r}\left(q_{i-1}+4 q_{i}+q_{i+1}\right)-G_{c}\left(s_{i-1}+4 s_{i}+s_{i+1}\right), \\
& \frac{6}{h^{2}} q_{i-1}-\frac{12}{h^{2}} q_{i}+\frac{6}{h^{2}} q_{i+1}+2(i h+c) \operatorname{Pr}\left(-\frac{3}{h} q_{i-1}+\frac{3}{h} q_{i+1}\right) \\
& =-R a\left(\begin{array}{l}
3\left(N+q_{i-1}+4 q_{i}+q_{i+1}\right)^{2}\left(-\frac{3}{h} q_{i-1}+\frac{3}{h} q_{i+1}\right)^{2} \\
+\left(N+q_{i-1}+4 q_{i}+q_{i+1}\right)^{3}\left(\frac{6}{h^{2}} q_{i-1}-\frac{12}{h^{2}} q_{i}+\frac{6}{h^{2}} q_{i+1}\right)
\end{array}\right), \\
& \frac{6}{h^{2}} s_{i-1}-\frac{12}{h^{2}} s_{i}+\frac{6}{h^{2}} s_{i+1}+2(i h+c) S c\left(-\frac{3}{h} s_{i-1}+\frac{3}{h} s_{i+1}\right)=0,
\end{aligned}
$$

with the boundary conditions

$$
\begin{aligned}
& d_{-1}+4 d_{0}+d_{1}=q_{-1}+4 q_{0}+q_{1}=s_{-1}+4 s_{0}+s_{1}=1, \\
& d_{n-1}+4 d_{n}+d_{n+1}=q_{n-1}+4 q_{n}+q_{n+1}=s_{n-1}+4 s_{n}+s_{n+1}=0,
\end{aligned}
$$

Solving the system of nonlinear Eqs. (19)-(22), we have the values of $d_{i}, q_{i}$, and $S_{i}$ for $-1 \leq i \leq n+1$, and substituting in Eqs. (16)-(18), we find the velocity distributions $(f)$, the temperature distributions $(\theta)$, and the concentration distributions $(\Phi)$. 


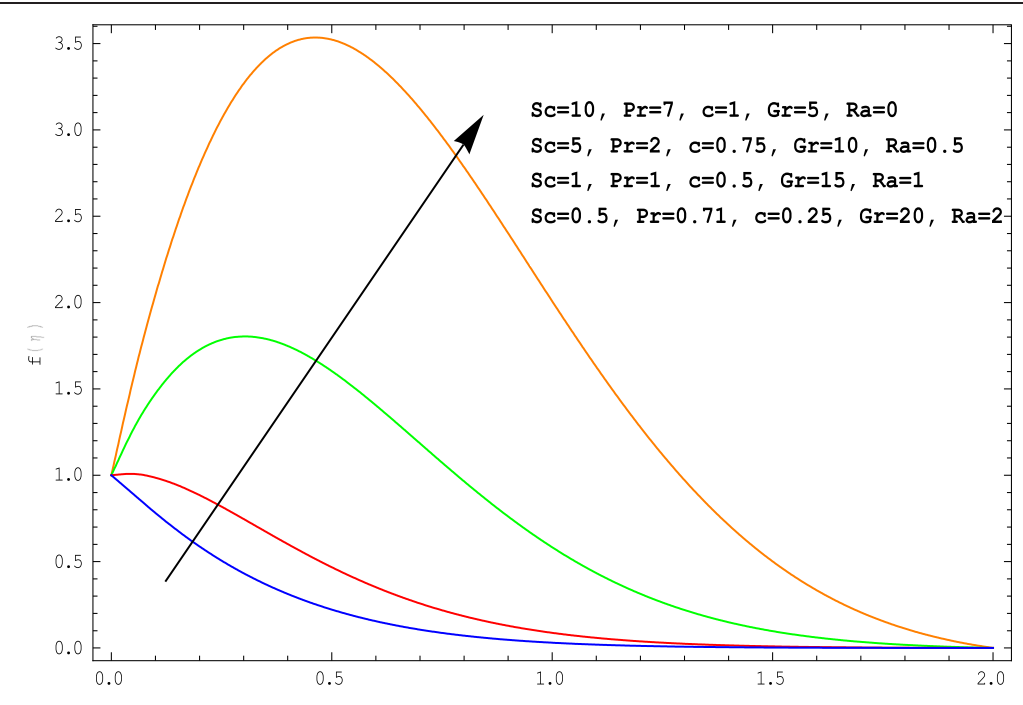

Fig. 2 Fluid velocity for different values of $S c, P r, C, G_{r}$ and $R a$

\section{Numerical results and discussion}

The problem of an unsteady free convection fluid flow past a moving vertical porous plate with thermal radiation and mass transfer has been computed for the velocity $(f)$, the temperature $(\theta)$, and the concentration $(\Phi)$ with boundary layer for different parameters as Prandtl number $(\operatorname{Pr})$, Schmidt number $(S c)$, thermal Grashof number $\left(G_{r}\right)$, solutal Grashof number $\left(G_{c}\right)$, the temperature difference parameter $(N)$, radiation parameter $(R a)$, and suction parameter $(c)$ with step size $\Delta \eta=0.05$. In this study, we consider the following values: $\operatorname{Pr}=0.71, \ldots, 7.0, S c=0.5, \ldots, 5.0, G_{r}=5, \ldots, 20, G_{c}=5, \ldots, 20$, $N=0.1, R a=0,0.1, \ldots, 2.0$ and $c=0.25, \ldots, 1.0$. In Figs. 2,3 , and 4 , we observe an enhancement the velocity, temperature, and concentration distributions in the fluid according to above different values.

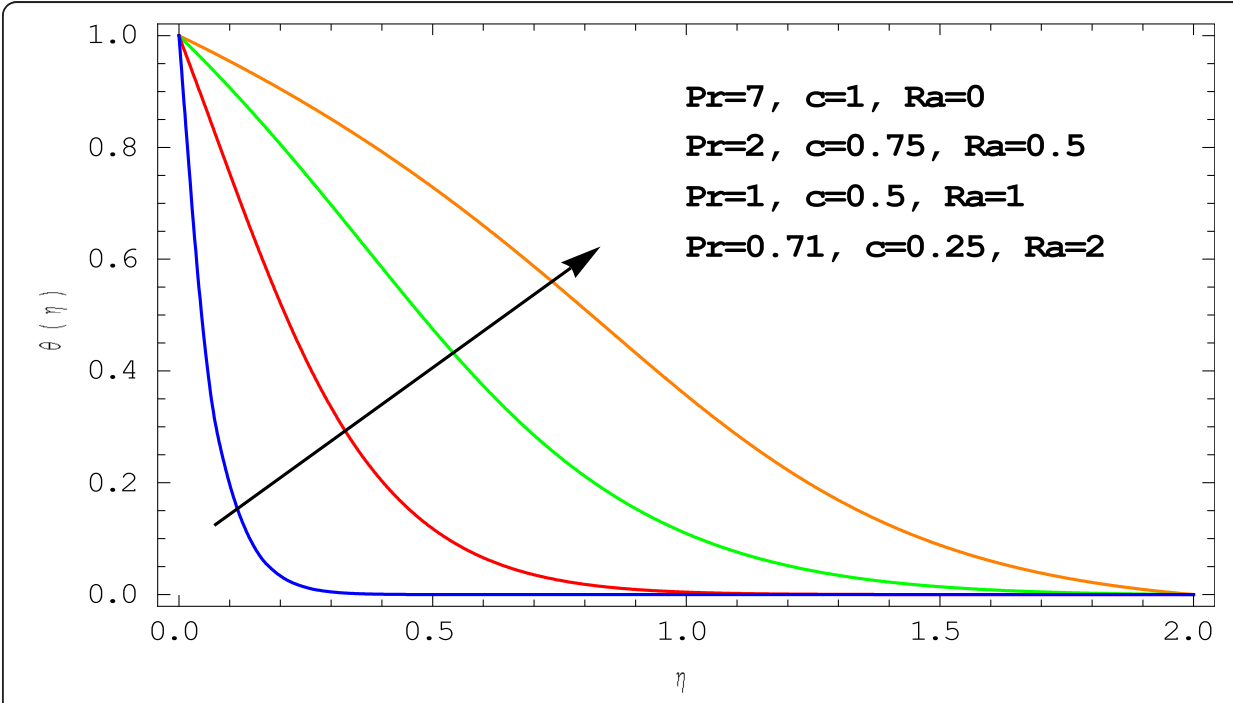

Fig. 3 Fluid temperature for different values of $\operatorname{Pr}, c$, and $R a$ 


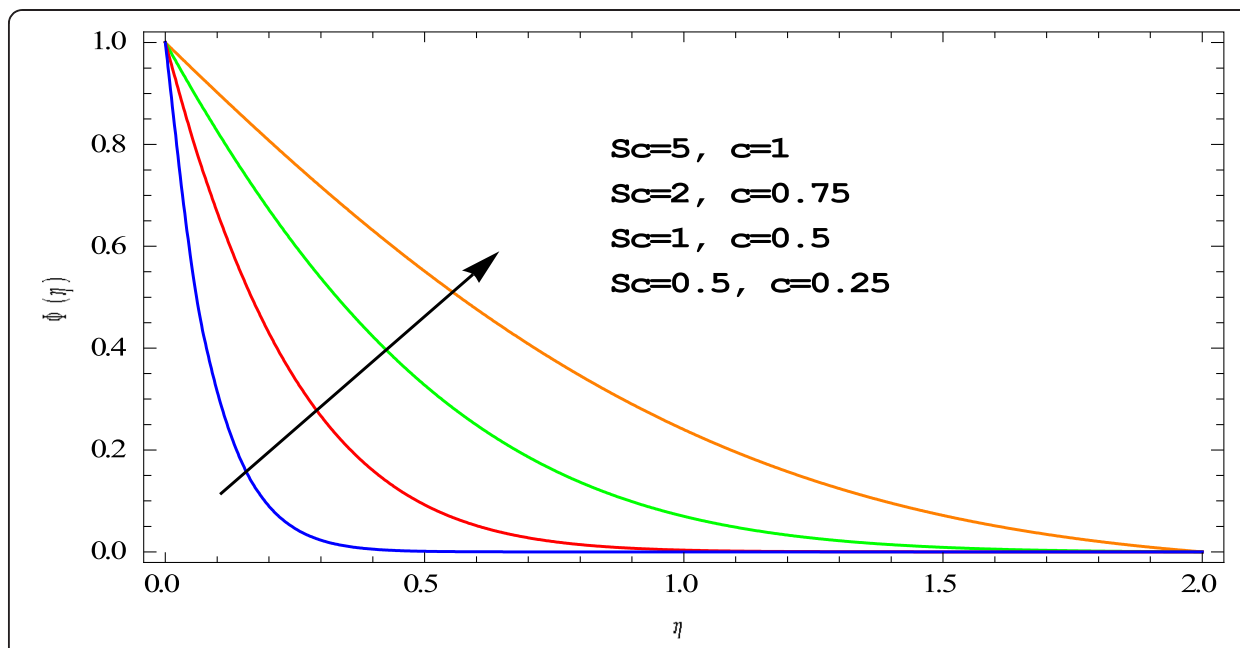

Fig. 4 Fluid concentration for different values of Sc and $c$

In Fig. 2, we observe the fluid velocity increased and after a very short distance from the plate reach to its maximum value and thereafter find a gradual decrease in the fluid velocity to zero. The fluid velocity enhances when there is a decrease in Prandtl number $(\operatorname{Pr})$, Schmidt number $(S c)$, and suction parameter $(c)$, although an increase in the thermal Grashof number $\left(G_{r}\right)$, solutal Grashof number $\left(G_{c}\right)$, and radiation parameter $(R a)$ will enhance the fluid velocity.

Figure 3 shows the fluid temperature decreased gradually to zero after some distance away from the plate. It observes that as an increase Prandtl number (Pr) and suction parameter $(c)$ is a major decrease in the fluid temperature, while an increase radiation parameter $(R a)$ will enhance the fluid temperature.

Figure 4 shows the fluid concentration is maximum at the plate surface and decreases until vanishes at some distance away from the plate. It is noted that a decrease in

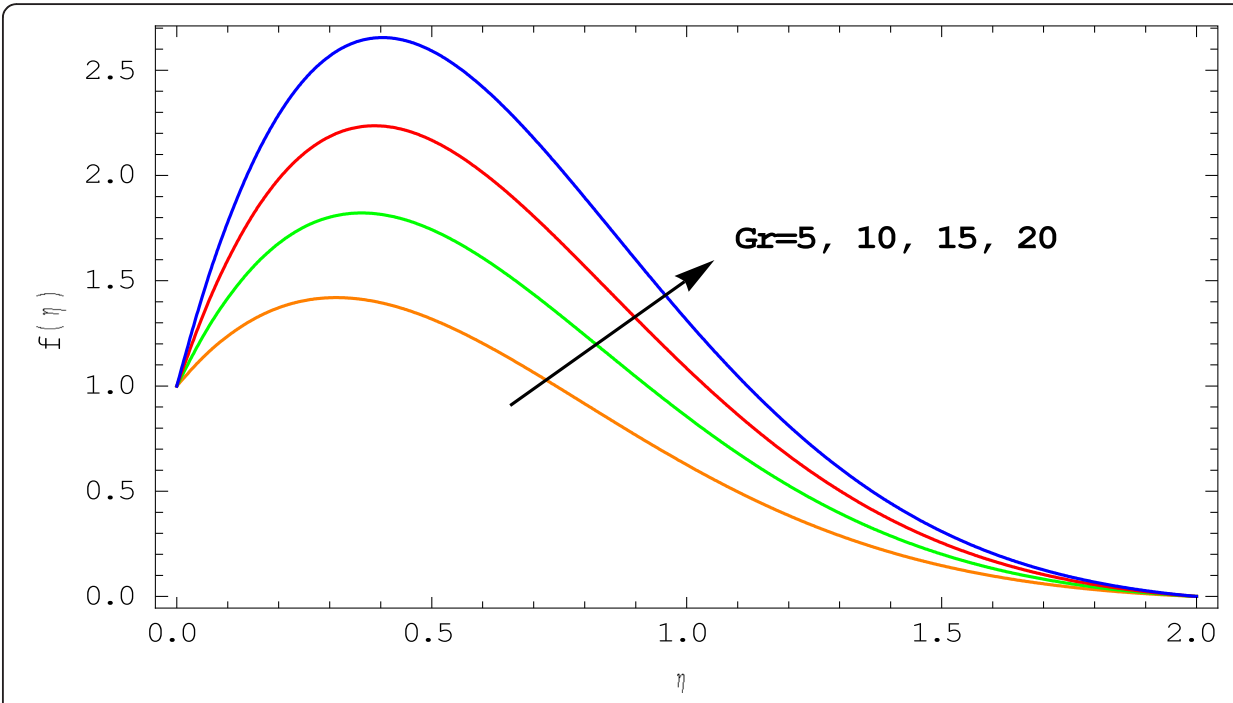

Fig. 5 Fluid velocity with increasing of the thermal Grashof number $\left(G_{r}\right)$ 


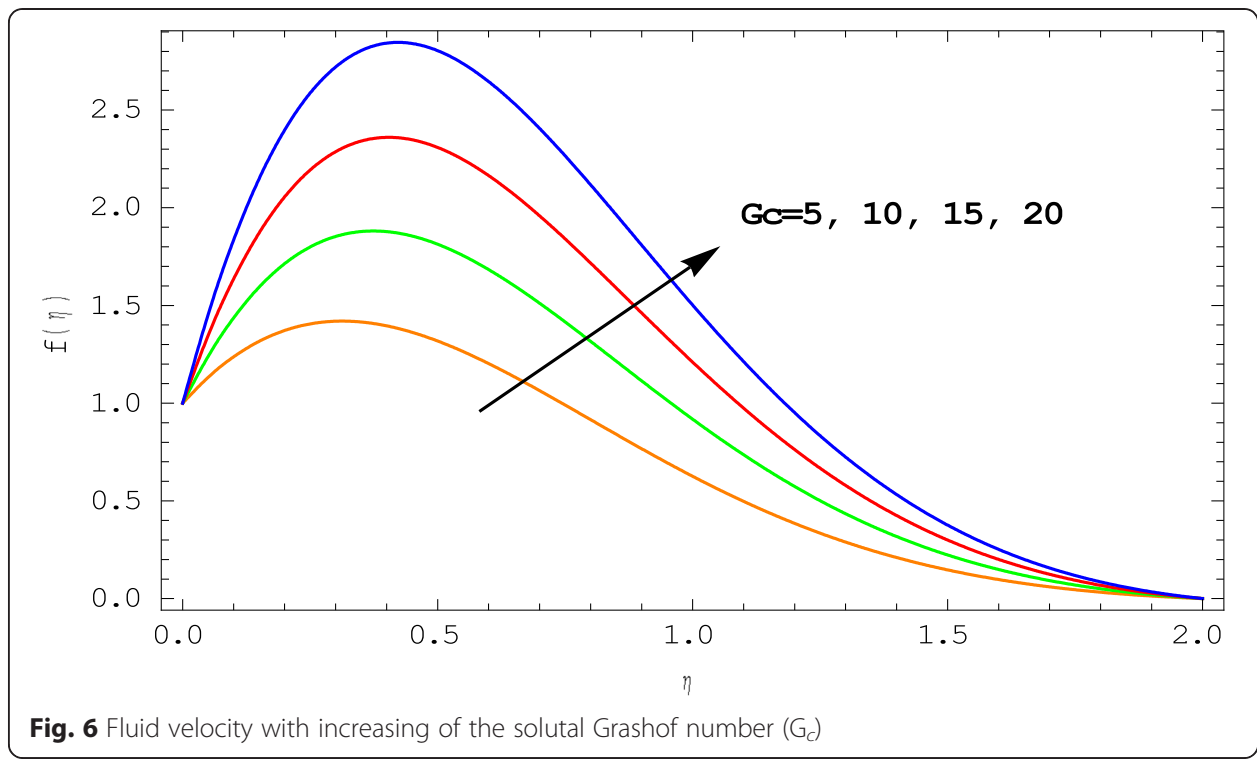

Schmidt number $(S c)$ and suction parameter $(c)$ will consolidate the fluid concentration.

Also, we investigated the distributions of the fluid velocity, the fluid temperature, and the fluid concentration for following default values: $G_{r}=5, G_{c}=5, \operatorname{Pr}=0.71, S c=0.5$, $R a=0.1, c=0.25$, and $N=0.1$.

Figures 5 and 6 show fluid velocity distributions for different values of thermal Grashof number $\left(G_{r}\right)$ and solutal Grashof number $\left(G_{c}\right)$. The velocity enhances (increases) with an increase in thermal Grashof number $\left(\mathrm{G}_{r}\right)$ and solutal Grashof number $\left(\mathrm{G}_{c}\right)$. It is shown that the peak values of the fluid velocity increase near the plate rapidly and then decay smoothly to the initial velocity (zero). This is due to the fact that buoyancy force enhances fluid velocity and increases the boundary layer thickness with an increase in the value of $\mathrm{G}_{r}$ or $\mathrm{G}_{c}$.

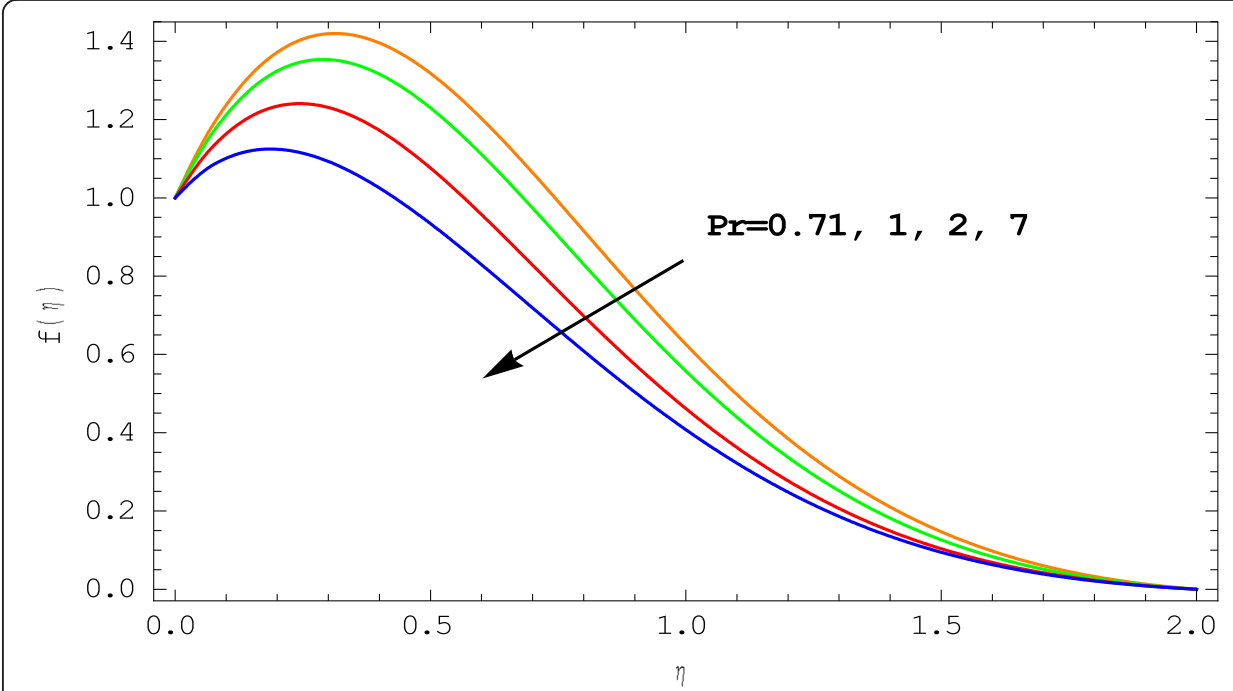

Fig. 7 Fluid velocity with increasing of the Prandtl number (Pr) 


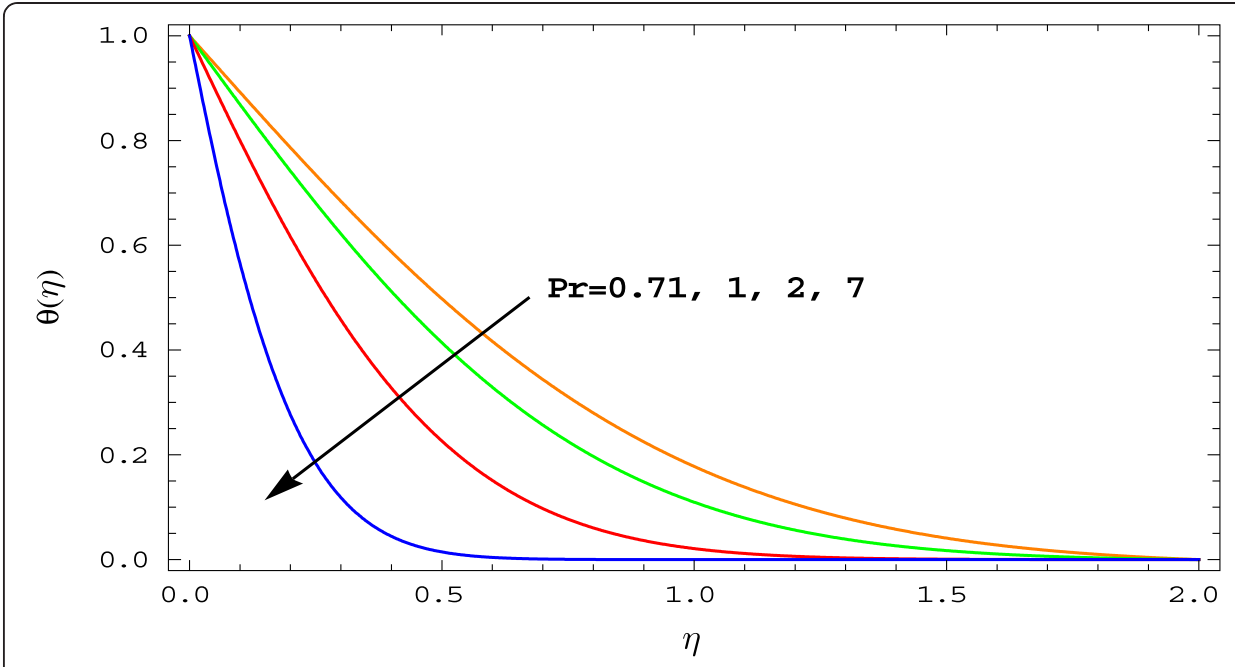

Fig. 8 Fluid temperature with increasing of the Prandtl number (Pr)

The influence of the Prandtl number ( $\operatorname{Pr})$ on the fluid velocity and the fluid temperature distributions is shown in Figs. 7 and 8 respectively. It is noted that an increase in the Prandtl number $(\operatorname{Pr})$ results in a decrease of the velocity and a decrease of the temperature which using smaller values of $(\operatorname{Pr})$ causes the boundary layer to be thicker reducing the rate of heat transfer and increasing the thermal conductivity; therefore, heat is able to diffuse away from the heated surface more rapidly than for higher values of $(\mathrm{Pr})$.

The influence of the Schmidt number $(S c)$ on the fluid velocity and the fluid concentration distributions is shown in Figs. 9 and 10 respectively. It is observed that an increase in the Schmidt number $(S c)$ results in a decrease of the velocity and a decrease of the concentration. Physically, the increase of $(S c)$ means a decrease of molecular diffusion $(D)$, which results in a decrease in the concentration boundary layer.

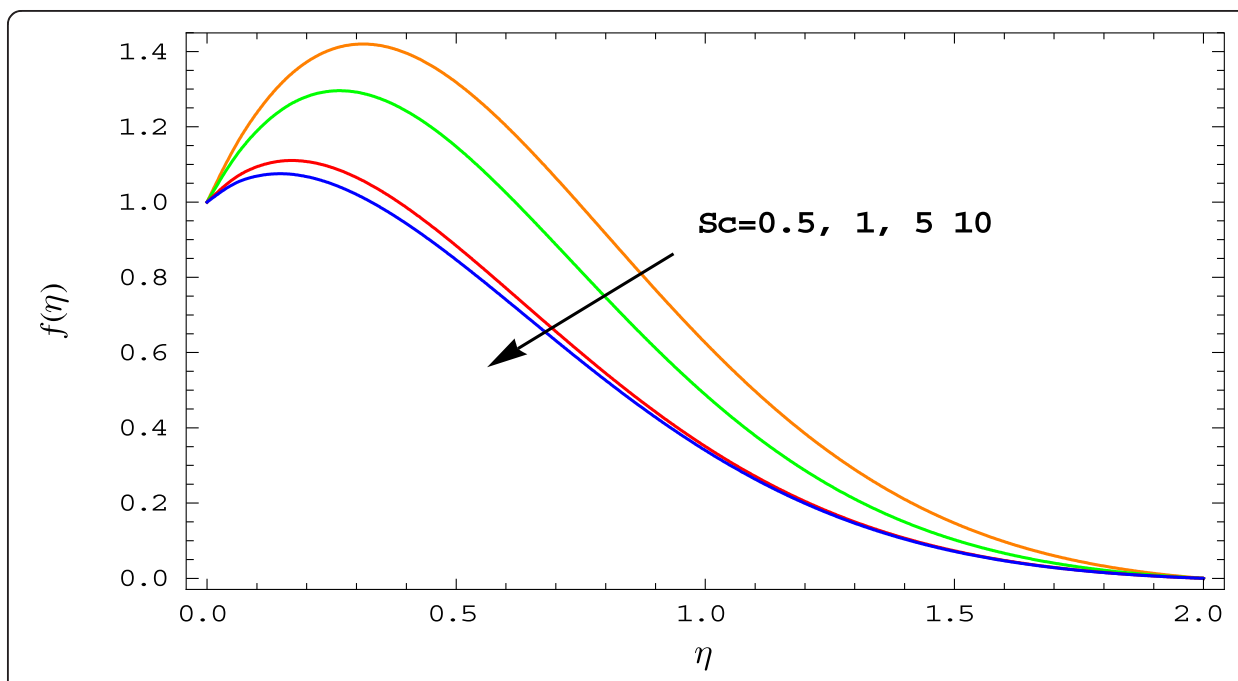

Fig. 9 Fluid velocity with increasing of the Schmidt number (SC) 


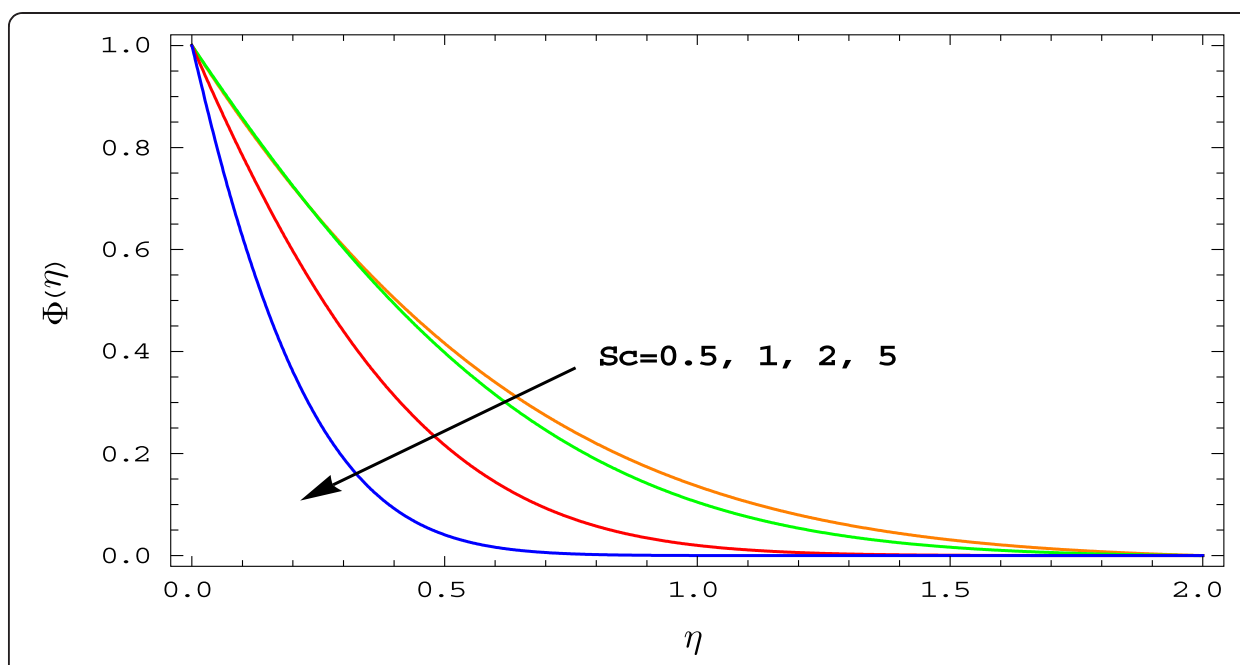

Fig. 10 Fluid concentration with increasing of the Schmidt number (Sc)

At different values of the suction parameter $(c)$, it is illustrated that a decrease in the fluid velocity, the fluid temperature, and the fluid concentration occurs when there is an increase in the suction parameter $(c)$ as shown in Figs. 11, 12, and 13. This is due to that the suction parameter retards the velocity of the flow field.

Figures 14 and 15 show the influence of the thermal radiation parameter $(\mathrm{R} a)$ on the fluid velocity and the fluid temperature, which an increase of the thermal radiation parameter $(R a)$ results in an increase of the velocity and temperature within the boundary layer. This is due to the fact that an increase in the value of $(R a)$ implies increasing of thermal radiation in the thermal boundary layer which results in an increase in the value of the temperature profile in the thermal boundary layer.

Now, it is necessary to interest some physical quantities, which are important characteristics of fluid flow, rate of heat transfer, and rate of mass transfer:

(i) The local shear wall Stress $\tau_{w}$ is given by

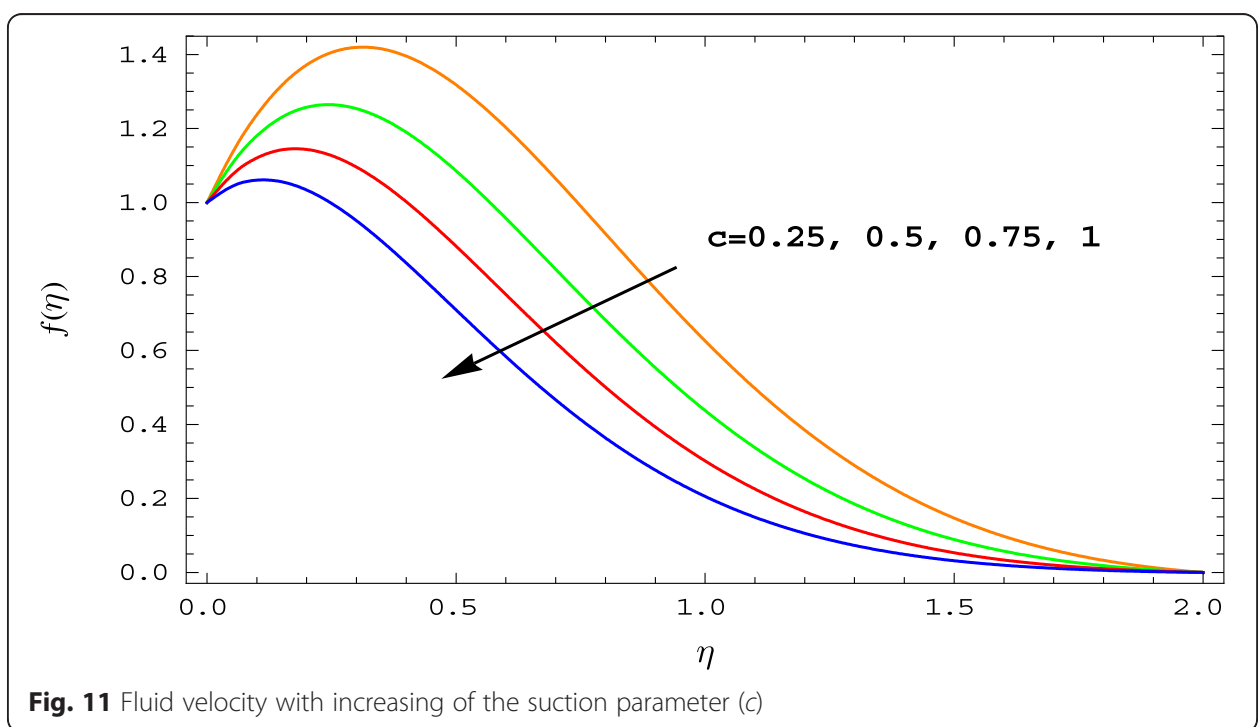




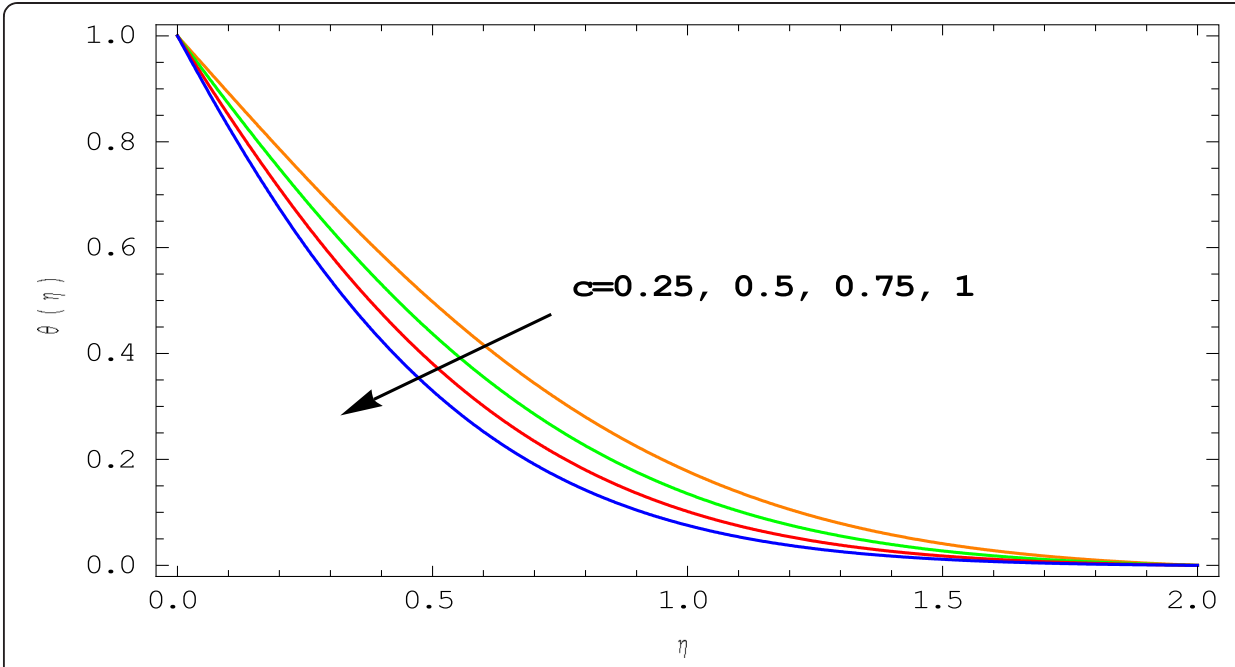

Fig. 12 Fluid temperature with increasing of the suction parameter (c)

$$
\tau_{w}=\rho v\left(\frac{\partial u}{\partial y}\right)_{y=0}
$$

and the dimensionless of local shear wall stress (the local skin friction $H$ )

$$
H=\frac{2 t \tau_{w}}{\rho U \sqrt{v t}}=f^{\prime}(0),
$$

Substituting Eq. (16) in Eq. (24), we obtain

$$
H=d_{-1}+4 d_{0}+d_{1}
$$

(ii) The local surface heat flux $q_{w}$ is given by

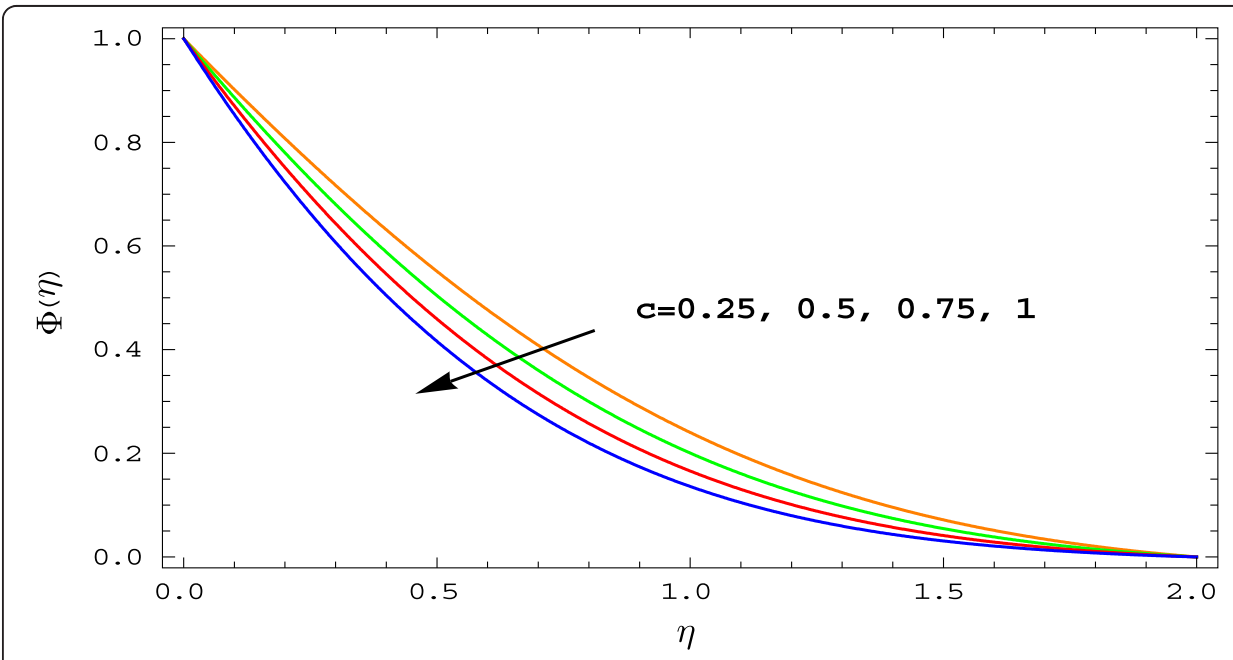

Fig. 13 Fluid concentration with increasing of the suction parameter (c) 


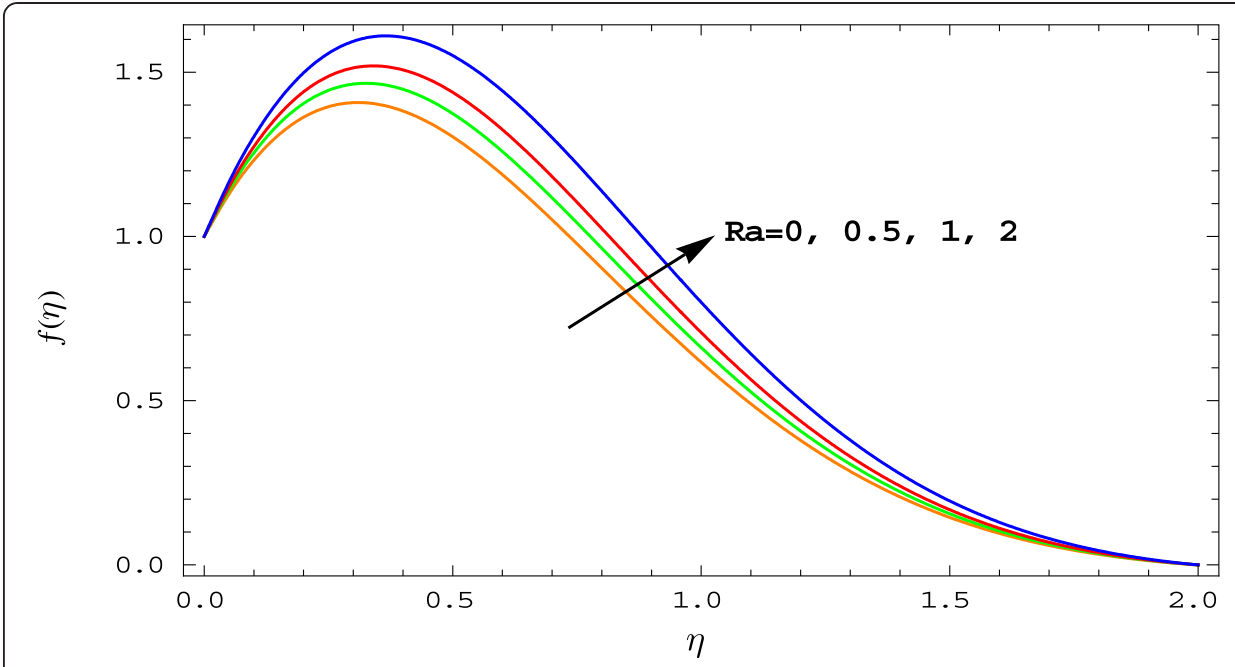

Fig. 14 Fluid velocity with increasing of the radiation parameter $(R a)$

$$
q_{w}=-k\left(\frac{\partial T}{\partial y}\right)_{y=0}-\frac{4 \sigma}{3 \delta}\left(\frac{\partial T^{4}}{\partial y}\right)_{y=0}
$$

and the dimensionless of the local surface heat flux (the local Nusselt number $\mathrm{Nu}$ )

$$
N u=\frac{2 q_{w} \sqrt{v t}}{k\left(T_{w}-T_{\infty}\right)}=-\theta^{\prime}(0)\left[1+R a(\theta(0)+N)^{3}\right],
$$

Substituting Eq. (17) in Eq. (27), we obtain

$$
N u=\frac{3}{h}\left(q_{-1}-q_{1}\right)\left[1+R a\left(q_{-1}+4 q_{0}+q_{1}+N\right)^{3}\right]
$$

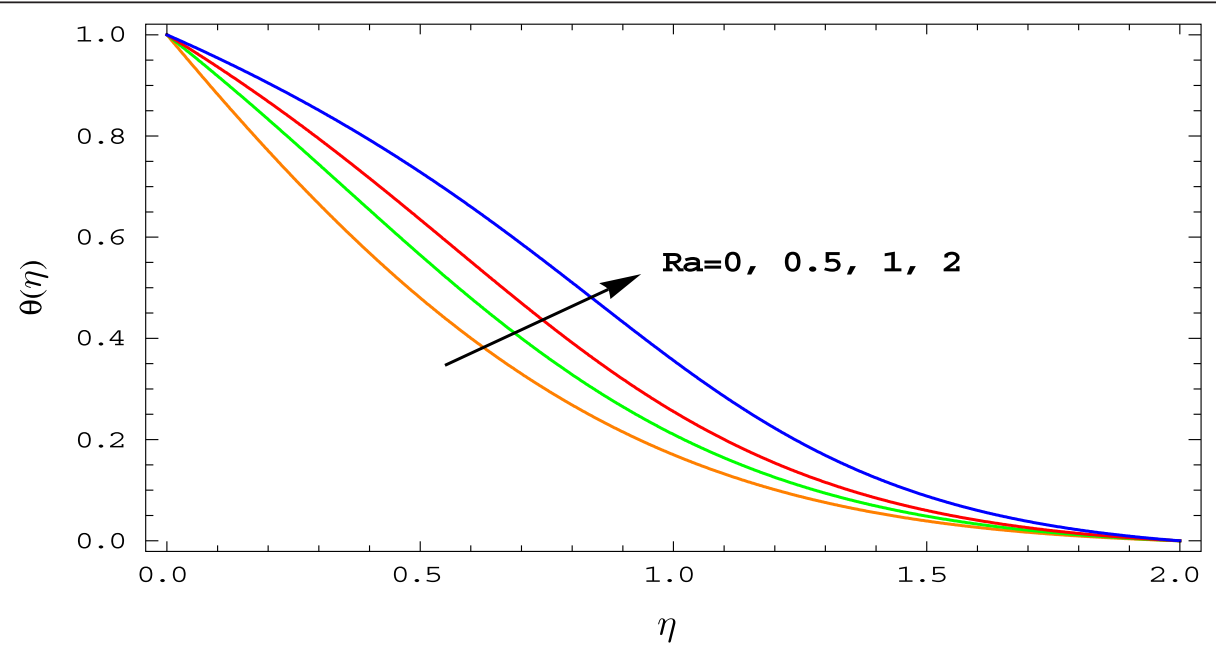

Fig. 15 Fluid temperature with increasing of the radiation parameter $(R a)$ 
Table 2 Values of skin friction $(H)$, Nusselt number $(\mathrm{Nu})$, and Sherwood number $(\mathrm{Mu})$ for different values of $G_{r}$

\begin{tabular}{llll}
\hline$G_{r}$ & $H$ & $N u$ & $M u$ \\
\hline 1 & 1.2463919077267462 & 1.2231565308893466 & 0.9936352340378196 \\
2 & 1.6660933568936933 & 1.2231565308893466 & 0.9936352340378196 \\
3 & 2.0857948060606404 & 1.2231565308893466 & 0.9936352340378196 \\
4 & 2.505496255227584 & 1.2231565308893466 & 0.9936352340378196 \\
5 & 2.9251977043945327 & 1.2231565308893466 & 0.9936352340378196 \\
10 & 5.0237049502292574 & 1.2231565308893466 & 0.9936352340378196 \\
15 & 7.122212196063995 & 1.2231565308893466 & 0.9936352340378196 \\
20 & 9.220719441898726 & 1.2231565308893466 & 0.9936352340378196 \\
\hline
\end{tabular}

(iii) The local surface mass flux $S_{w}$ is given by

$$
S_{w}=-D\left(\frac{\partial C}{\partial y}\right)_{y=0}
$$

and the dimensionless of the local surface mass flux (the local Sherwood number $M u)$

$$
M u=\frac{2 S_{w} \sqrt{v t}}{D\left(C_{w}-C_{\infty}\right)}=-\Phi^{\prime}(0),
$$

Substituting Eq. (18) in Eq. (30), we obtain

$$
M u=-\left(s_{-1}+4 s_{0}+s_{1}\right)
$$

So, we calculate the physical quantities, the local shear wall stress, the local surface heat flux, and the local surface mass flux for the following default values:

$$
G_{r}=5, G_{c}=5, \operatorname{Pr}=0.71, S c=0.5, R a=0.1, c=0.25 \text { and } N=0.1
$$

Tables 2 and 3 show an increase in skin friction $(H)$; meanwhile, Nusselt number $(\mathrm{Nu})$ and Sherwood number $(\mathrm{Mu})$ are not influenced with an increase in thermal Grashof number $\left(G_{r}\right)$ and solutal Grashof number $\left(G_{c}\right)$ due to buoyancy effects.

Table 3 Values of skin friction $(\mathrm{H})$, Nusselt number $(\mathrm{Nu})$, and Sherwood number $(\mathrm{Mu})$ for different values of $G_{c}$

\begin{tabular}{llll}
\hline$G_{C}$ & $H$ & $N u$ & $M u$ \\
\hline 1 & 1.090082737814507 & 1.2231565308893466 & 0.9936352340378196 \\
2 & 1.5488614794595108 & 1.2231565308893466 & 0.9936352340378196 \\
3 & 2.00764022110452 & 1.2231565308893466 & 0.9936352340378196 \\
4 & 2.466418962749522 & 1.2231565308893466 & 0.9936352340378196 \\
5 & 2.9251977043945327 & 1.2231565308893466 & 0.9936352340378196 \\
10 & 5.219091412619557 & 1.2231565308893466 & 0.9936352340378196 \\
15 & 7.512985120844588 & 1.2231565308893466 & 0.9936352340378196 \\
20 & 9.806878829069618 & 1.2231565308893466 & 0.9936352340378196 \\
\hline
\end{tabular}


Table 4 Values of skin friction $(\mathrm{H})$, Nusselt number $(\mathrm{Nu})$, and Sherwood number $(\mathrm{Mu})$ for different values of $\mathrm{Pr}$

\begin{tabular}{llll}
\hline $\operatorname{Pr}$ & $H$ & $\mathrm{Nu}$ & $\mathrm{Mu}$ \\
\hline 0.5 & 3.1748494490032115 & 1.0152949217878635 & 0.9936352340378196 \\
0.6 & 3.048801108244991 & 1.1152909590540125 & 0.9936352340378196 \\
0.7 & 2.9358207733605752 & 1.21345346885328224 & 0.9936352340378196 \\
0.8 & 2.8347301277348027 & 1.3095074845586798 & 0.9936352340378196 \\
0.9 & 2.7442306329099466 & 1.403355089091998 & 0.9936352340378196 \\
1.0 & 2.6630371830784103 & 1.495014187831923 & 0.9936352340378196 \\
5.0 & 1.6438175915395838 & 4.341215116765501 & 0.9936352340378196 \\
7.0 & 1.4967848342088548 & 5.546218261092864 & 0.9936352340378196 \\
\hline
\end{tabular}

Table 4 shows that an increase in Prandtl number $(\mathrm{Pr})$ results in a decrease in skin friction $(H)$ and an increase in Nusselt number $(\mathrm{Nu})$, while Sherwood number $(\mathrm{Mu})$ is not influenced. The reason for this is that smaller values of (Pr) are equivalent to increasing thermal conductivities, and therefore, heat is able to diffuse away from the heated plate more rapidly than for higher values of $(\mathrm{Pr})$.

Also, Table 5 evidences that an increase in Schmidt number $\left(S_{c}\right)$ results in a decrease in skin friction $(H)$ and an increase in Sherwood number $(M u)$, while Nusselt number $(\mathrm{Nu})$ is not affected, because the velocity and species concentration and the boundary layer thickness decrease.

An increase in thermal radiation parameter $(R a)$ results in an enhancement in skin friction $(H)$ and Nusselt number $(N u)$ and is not affected in Sherwood number $(M u)$ as shown in Table 6. Higher values of radiation parameter $(R a)$ imply higher values of wall temperature.

At various values of the suction parameter $(c)$, we obtain an increase in Nusselt number $(N u)$ and Sherwood number $(M u)$ and a decrease in skin friction $(H)$ due to an increase in the suction parameter $(c)$ which leads to a decrease in the velocity field as shown in Table 7.

Tables 8 and 9 show values of skin friction $(H)$, Nusselt number $(N u)$, and Sherwood number $(M u)$ for different values of radiation parameter $(R a)$, suction parameter $(c)$, and step size $\Delta \eta=0.08$, which we compare our method with Makinde [8].

Table 5 Values of skin friction $(H)$, Nusselt number $(\mathrm{Nu})$, and Sherwood number $(\mathrm{Mu})$ for different values of SC

\begin{tabular}{llll}
\hline SC & $H$ & $\mathrm{Nu}$ & $\mathrm{Mu}$ \\
\hline 0.5 & 2.9251977043945327 & 1.2231565308893466 & 0.9936352340378196 \\
0.6 & 2.8007296377108073 & 1.2231565308893466 & 1.0924002447788919 \\
0.7 & 2.6894329744287013 & 1.2231565308893466 & 1.1893023675501322 \\
0.8 & 2.5900241032965514 & 1.2231565308893466 & 1.2841027147279753 \\
0.9 & 2.501146004684635 & 1.2231565308893466 & 1.376726015953622 \\
1.0 & 2.4214847149694236 & 1.2231565308893466 & 1.467203249665232 \\
5.0 & 1.4247245244892035 & 1.2231565308893466 & 4.296088707510315 \\
10.0 & 1.1514325570406 & 1.2231565308893466 & 7.245271189173215 \\
\hline
\end{tabular}


Table 6 Values of skin friction $(\mathrm{H})$, Nusselt number $(\mathrm{Nu})$, and Sherwood number $(\mathrm{Mu})$ for different values of $R a$

\begin{tabular}{llll}
\hline$R a$ & $H$ & $N u$ & $M u$ \\
\hline 0.0 & 2.874365896653261 & 1.1988792951798555 & 0.9936352340378196 \\
0.1 & 2.9251977043945327 & 1.2231565308893466 & 0.9936352340378196 \\
0.2 & 2.97389549938379 & 1.247030707762581 & 0.9936352340378196 \\
0.3 & 3.020655195438234 & 1.2704065679717307 & 0.9936352340378196 \\
0.4 & 3.0656367834279035 & 1.2932793420917474 & 0.9936352340378196 \\
0.5 & 3.108975622965138 & 1.3156728360303704 & 0.9936352340378196 \\
1.0 & 3.304672971623882 & 1.4215252909945666 & 0.9936352340378196 \\
\hline
\end{tabular}

\section{Conclusion}

In this paper, we presented a numerical study using cubic B-spline method which transforms the equations governing the fluid flow to system of nonlinear algebraic equations. We investigated the effects of thermal radiation and mass transfer on an unsteady free convection flow over a moving vertical porous plate. However, from computational results, an increase in the thermal radiation results in an increase in the fluid velocity and temperature profiles. The rate of mass transfer is not affected which increases most parameters except at an increase Schmidt number and suction parameter the rate of mass transfer is enhanced. Numerical results show that the proposed method demonstrates efficient solutions of the considered problem and similar to Makinde [9] as in Tables 8 and 9.

\section{Nomenclature}

$u$ The velocity component along the $x$ direction

$v$ The velocity component along the $y$ direction

$v$ The kinematic viscosity

$g$ The acceleration due to gravity

$T$ The temperature

$C$ The concentration

$D$ The coefficient of mass diffusivity

$\alpha$ The thermal diffusivity

Table 7 Values of skin friction $(H)$, Nusselt number $(\mathrm{Nu})$, and Sherwood number $(\mathrm{Mu})$ for different values of $c$

\begin{tabular}{llll}
\hline$c$ & $H$ & $N u$ & $M u$ \\
\hline 0.15 & 3.1180597384267905 & 1.1285940213795056 & 0.9288763615384958 \\
0.25 & 2.9251977043945327 & 1.2231565308893466 & 0.9936352340378196 \\
0.35 & 2.7184241349431986 & 1.3210207458453906 & 1.06044396795560666 \\
0.45 & 2.49970119301641 & 1.4219720321982816 & 1.1292086423019558 \\
0.50 & 2.3864398144757857 & 1.4735387634381403 & 1.164294550801099 \\
0.75 & 1.789495603232492 & 1.7412527134729352 & 1.34627768447708 \\
1.00 & 1.1575700732394765 & 2.023232554446144 & 1.53809626000003407 \\
1.25 & 0.5075519675894924 & 2.316808917611598 & 1.7383411900501997 \\
\hline
\end{tabular}


Table 8 Comparison of our method and Makinde [8] using different values of Ra and step size $\Delta \eta=0.08$

\begin{tabular}{llllllll}
\hline$R a$ & Our method & & & & Ref. [8] & \\
& $H$ & Nu & Mu & & $H$ & Nu & Mu \\
\hline 0.1 & 3.0956482 & 1.2106281 & 0.96378847 & & 3.0377686 & 1.1188872 & 0.9635597 \\
0.3 & 3.1946891 & 1.2570321 & 0.96378847 & & 3.1898811 & 1.2583769 & 0.9635597 \\
0.5 & 3.2867125 & 1.3013334 & 0.96378847 & & 3.2823720 & 1.3020452 & 0.9635597 \\
1.0 & 3.4926218 & 1.4037436 & 0.96378847 & & 3.4892859 & 1.4037339 & 0.9635597 \\
\hline
\end{tabular}

$k$ The thermal conductivity

$q_{r}$ The local radiative heat flux

$\beta$ The volumetric expansion coefficient due to temperature

$\lambda$ The volumetric expansion coefficient due to concentration

$T_{w}$ The uniform temperature of the fluid at the plate

$C_{w}$ The uniform concentration of the fluid at the plate

$T_{\infty}$ The temperature of the fluid far away from the plate

$C_{\infty}$ The concentration of the fluid far away from the plate

$U$ The plate characteristic velocity

$\sigma$ The Stefan-Boltzmann constant

$\delta$ The mean absorption coefficient

$\eta$ The dimensionless similarity variable

$G_{r}$ and $G_{c}$ The local free convection parameters

Pr The Prandtl number

$\mathrm{Ra}$ The radiation parameter

$N$ The temperature difference parameter

Sc The Schmidt number

$f$ The velocity distributions

$\theta$ The temperature distributions

$\Phi$ The concentration distributions

$\tau_{w}$ The local shear wall stress

$H$ The local skin friction

$q_{w}$ The local surface heat flux

$\mathrm{NuThe}$ local Nusselt number

$S_{w}$ The local surface mass flux

$M u$ The local Sherwood number

Table 9 Comparison of our method and Makinde [8] using different values of $c$ and step size $\Delta \eta=0.08$

\begin{tabular}{|c|c|c|c|c|c|c|}
\hline \multirow[t]{2}{*}{ c } & \multicolumn{3}{|c|}{ Our method } & \multicolumn{3}{|l|}{ Ref. [8] } \\
\hline & H & $\mathrm{Nu}$ & $\mathrm{Mu}$ & $H$ & $\mathrm{Nu}$ & $\mathrm{Mu}$ \\
\hline 0.15 & 3.3111497 & 1.1142168 & 0.8961870 & 3.3082627 & 1.1160080 & 0.8957656 \\
\hline 0.35 & 2.8683900 & 1.3101159 & 1.0333192 & 2.8608264 & 1.3123583 & 1.0332379 \\
\hline 0.75 & 1.8784393 & 1.7347141 & 1.3285810 & 1.8613571 & 1.7382843 & 1.3287779 \\
\hline 1.25 & 0.5562163 & 2.3119201 & 1.7290747 & 0.5299713 & 2.3180810 & 1.7288166 \\
\hline
\end{tabular}




\section{Acknowledgements}

The authors are grateful and express sincere thanks to the reviewers for their detailed reading, precious proposals, and comments

\section{Authors' contributions}

The authors read and approved the final manuscript.

\section{Funding}

The authors declare that they had no funding.

\section{Availability of data and materials}

Not applicable.

\section{Competing interests}

The authors declare that they have no competing interests.

\section{Author details}

${ }^{1}$ Basic Science Department, Higher Technological Institute, Tenth of Ramadan City, Egypt. ${ }^{2}$ Mathematics Department, Faculty of Science, Al-Azhar University, Cairo, Egypt.

Received: 19 May 2019 Accepted: 25 July 2019

\section{Published online: 04 October 2019}

\section{References}

1. Mohammadein, A.A., El-Shaer, N.A.: Influence of variable permeability on combined free and forced convection flow past a semi-infinite vertical plate in a saturated porous medium. Heat Mass Transfer. 40, 341-346 (2004)

2. Zhang, J., Subotic, M., Lai, F.C.: Transient and steady natural convection from a heat source embedded in thermally stratified porous layer. Int. J. Thermal Sci. 49(9), 1527-1535 (2010)

3. Alam, M.S., Rahman, M.M., Maleque, M.A., Ferdows, M.: Dufour and Soret effects on steady MHD combined free-forced convective and mass transfer flow past a semi-infinite vertical plate. Thammasat Int. J. Sci. Tech. 11(2), 1-12 (2006)

4. Brewster, M.Q:: Thermal Radiative Transfer and Properties. John Wiley and Sons, New York (1992)

5. Bestman, A.R.: Natural convection boundary layer with suction and mass transfer in a porous medium. Int. J. Energy Res. 14, 389-396 (1990)

6. Singh, A.K., Soundalgekar, V.M.: Transient free convection in cold water past an infinite porous plate. Int. J. Energy Res. 14(4), 413-420 (1990)

7. Hossain, M.A.: Effect of transpiration on combined heat and mass transfer in mixed convection along a vertical plate. Int. J. Energy Res. 16, 761-769 (1992)

8. Makinde, O.D.: Free convection flow with thermal radiation and mass transfer past a moving vertical porous plate. International Communications in Heat and Mass Transfer. 32, 1411-1419 (2005)

9. Reddy, G.V.R., Reddy, N.B., Gorla, R.S.R.: Radiation and chemical reaction effects on MHD flow along a moving vertical porous plate. Int. J. of Applied Mechanics and Engineering. 21(1), 157-168 (2016)

10. Reddy, M.J., Reddy, J., Sivaiah, G.: Mass transfer effects on MHD unsteady free convective Walter's memory flow with constant suction and heat sink through porous media. Adv. Appl. Res. 3(2), 1137-1143 (2012)

11. Pal, D., Chatterjee, S.: Heat and mass transfer in MHD non-Darcian flow of a micropolar fluid over a stretching sheet embedded in a porous media with non-uniform heat source and thermal radiation. Commun Nonlinear Sci Numer Simulat. 15(7), 1843-1857 (2010)

12. Sharma, R.P., Makinde, O.D., Animasaun, I.L.: Buoyancy effects on MHD unsteady convection of a radiating chemically reacting fluid past a moving porous vertical plate in a binary mixture. Defect and Diffusion Forum. 387, 308-318 (2018)

13. Makinde, O.D., Olanrewaju, P.O., Charles, W.M.: Unsteady convection with chemical reaction and radiative heat transfer past a flat porous plate moving through a binary mixture. Afrika Mathematika. 22, 65-78 (2011)

14. Sajid, T., Sagheer, M., Hussain, S., Bilal, M.: Darcy-Forchheimer flow of Maxwell nanofluid flow with nonlinear thermal radiation and activation energy. AIP Adv. 8, 0351021-0351019 (2018)

15. Makinde, O.D., Olanrewaju, P.O.: Unsteady mixed convection with Soret and Dufour effects past a porous plate moving through a binary mixture of chemically reacting fluid. Chemical Engineering Communications. 198(7), 920-938 (2011)

16. Makinde, O.D., Ogulu, A.: The effect of thermal radiation on the heat and mass transfer flow of a variable viscosity fluid past a vertical porous plate permeated by a transverse magnetic field. Chemical Engineering Communications. 195(12), 1575-1584 (2008)

17. Ogulu, A., Makinde, O.D.: Unsteady hydromagnetic free convection flow of a dissipative and radiating fluid past a vertical plate with constant heat flux. Chemical Engineering Communications. 196(4), 454-462 (2009)

18. Caglar, N., Caglar, H.: B-spline method for solving linear system of second-order boundary value problems. Comput Math Appl. 57(5), 757-762 (2009)

\section{Publisher's Note}

Springer Nature remains neutral with regard to jurisdictional claims in published maps and institutional affiliations. 